\title{
Determinação da dose ótima de cloridrato de benzocaína na anestesia de tilápias (Oreochromis niloticus)
}

\section{Determination of the optimal dose of benzocaine hydrochloride in anesthesia of tilápia (Oreochromis niloticus)}

\author{
Marco Antonio da Rocha ${ }^{1}$; Carmen Esther Santos Grumadas ${ }^{2}$; \\ Edson Luis de Azambuja Ribeiro ${ }^{3 *}$; Ivone Yurika Mizubuti³; \\ Agostinho Ludovico ${ }^{4}$; Camila Constatino ${ }^{5}$
}

\begin{abstract}
Resumo
A anestesia em peixes é indicada para auxiliar a realização de diversos procedimentos como: captura, biometria, marcação, transporte, exame físico, procedimentos cirúrgicos e manejo reprodutivo. Neste experimento, determinou-se a dose de cloridrato de benzocaína na anestesia de tilápias (Oreochromis niloticus), com a realização de seis etapas com 240 peixes, sendo 40 peixes em cada etapa, em intervalo de dois meses. O peso médio e o (fator de condição alométrico) das tilápias em cada etapa foram de: 25,64 (2,5635); 167,58 (2,8776); 286,12 (2,5719); 388,24 (2,4955); 518,19 (2,8933); 592,71 (2,6750), respectivamente. $\mathrm{O}$ fator de condição alométrico mostrou que os animais utilizados no experimento estavam em boas condições $(\mathrm{P}>0,05)$. Em cada etapa, os peixes foram capturados e colocados em quatro recipientes contendo 5 litros de água com benzocaína diluída em $20 \mathrm{ml}$ de etanol, nas concentrações de 100, 140, 180 e $220 \mathrm{mg} /$ litro de água. O tempo de indução em segundos (TI) foi registrado para cada peixe, e após a indução anestésica foi realizada a biometria no tempo fixo de 10 minutos. Após, os peixes foram transferidos para recipientes contendo 20 litros de água com renovação constante para avaliar o tempo de recuperação em segundos (TR). Em cada etapa, foi calculada a dose mínima de benzocaína através do LRP (Linear Response Plateu) em um modelo que incluiu a dose de benzocaína (mg/l) e tempo de indução em segundos. Os valores do LRP foram respectivamente: 146,60 e 67,45; 155,95 e 76,$33 ; 160,45$ e 87,$42 ; 167,00$ e 108,$14 ; 165,87$ e 174,$03 ; 164,00$ e 139,80 . A dose ótima foi relacionada com o peso médio em cada fase resultando na equação: Dose $=149,65+0,03183 \times$ peso, $\left(r^{2}=0,73\right)$. Esta equação mostra que um aumento em $1 \mathrm{~g}$ no peso corporal corresponde a $0,032 \mathrm{mg} / \mathrm{l} \mathrm{na}$ dose de benzocaína.
\end{abstract}

Palavras-chave: Alometria, peixes, tempo de indução

\footnotetext{
Abstract

Fish anesthesia is indicated for several management procedures such as capturing, biometry, tagging, transporting, physical examination, reproductive management and surgical procedures. In this study the dose of benzocaine hydrochloride necessary for tilápia (Oreochromis niloticus) anesthesia was

1 Prof. Dr. Aposentado do Dept ${ }^{\circ}$ de Zootecnia, Universidade Estadual de Londrina, UEL, Londrina, PR. E-mail: marocw@hotmail. com

2 Prof ${ }^{\mathrm{a}}$. Dr ${ }^{\mathrm{a}}$. do Dept ${ }^{\mathrm{o}}$ de Clínicas Veterinárias, UEL, Londrina, PR. E-mail: grumadas@uel.br

3 Profs. Drs. do Dept ${ }^{\circ}$ de Zootecnia, UEL, Londrina, PR. Bolsista de Produtividade do CNPq. E-mail: mizubuti@uel.br; elar@uel. br

4 Prof. Dr. do Curso de Medicina Veterinária, Universidade do Norte do Paraná, UNOPAR, Arapongas, PR. E-mail: agostinho@, rocketmail.com

5 Doutoranda do Programa de Pós-Graduação em Ciência Animal, UEL, Londrina, PR. E-mail: caconstantino@hotmail.com

* Autor para correspondência 
determined through six phases with 240 fish. In each phase 40 fish were evaluated. The interval between phases was two months. Mean values for weight and (allometric condition factor), in each phase, were 25.64 (2.56), 167.58 (2.88), 286.12 (2.57), 388.24 (2.50), 518.19 (2.89), 592.71 (2.67), respectively. The values for allometric condition factors showed that the animals included in the experiments were in good body conditions $(\mathrm{P}>0.05)$. In each phase, fishes were captured and kept in four containers with five liters of water and benzocaine hydrochloride diluted in $20 \mathrm{~mL}$ of ethanol, in concentrations of 100, 140, 180 , and $220 \mathrm{mg} /$ liter of water. The time of induction in seconds (TI) was registered for each fish, and after the anesthetic induction the biometric analysis was conducted at fixed time of 10 minutes. After, the fishes were transferred to containers with 20 liters of water under constant flow, in order to evaluate the recovering time in seconds (TR). At each phase the minimum dose of benzocaine hydrochloride concentration was calculated using LRP (Linear Response Plateau). The model included the dose of benzocaine $(\mathrm{mg} / \mathrm{L})$ and the time of induction in seconds. The values of LRP were, respectively, 146.60 and $67.45,155.95$ and $76.33,160.45$ and $87.42,167.00$ and $108.14,165.87$ and $174.03,164.00$ and 139.80. The optimum dose was related to the mean weight in each phase, resulting in the equation: Dose $=149.65+0.03183 \mathrm{x}$ weigh $\left(\mathrm{r}^{2}=0.73\right)$. This equation showed that an increase of $1 \mathrm{~g}$ in the body weight corresponded to an increase of $0.032 \mathrm{mg} / \mathrm{L}$ in the dose of benzocaine hydrochloride.

Key words: Allometric, fish, induction time

\section{Introdução}

$\mathrm{Na}$ criação de peixes a anestesia é um procedimento para evitar o stress em diversos procedimentos como: marcação, captura, biometria, cirurgia, reprodução, transportes, entre outros. A via de administração é a inalação com o anestésico dissolvido em água (ROUBACH; GOMES, 2001).

A anestesia em peixes é dividida em seis estágios (THURMON; TRANQUILLI; BENSON, 1996). No estágio 1 - sedação leve - ocorre discreta perda da reação a estímulos externos visuais e táteis e equilíbrio normal. No estágio 2 - sedação profunda - ocorre discreta perda da reação a estímulos externos, exceto pressão intensa, aparece uma pequena redução na frequência opercular e o equilíbrio é normal. No estágio 3 - perda parcial do equilíbrio - há redução parcial do tono muscular, nado não sincronizado, aumento da frequência opercular e reação somente a estímulos táteis e vibratórios intensos. No estagio 4 - perda total do equilíbrio - verifica-se perda total do tônus muscular e equilíbrio, frequência opercular rápida e reação somente a estímulos de pressão intensa. No estagio 5 - perda da atividade reflexa - ocorre perda total da atividade reflexa, os movimentos operculares tornam-se muito superficiais e a frequência cardíaca diminui. No estágio 6 - colapso medular - cessam os movimentos operculares.
Bastos-Ramos, Gonçalves e Bacila (1998) citaram diversos anestésicos utilizados para peixes: MS-222, quinaldina, benzocaína, 2-phenoxiethanol, clobutanol, etomidato, metomidato, dióxido de carbono, nicotina, halotano, eugenol, thiopental, bicarbonato de sódio, eletronarcose, e outros. Para se avaliar a eficiência de um anestésico ele deve apresentar um tempo de indução inferior a três minutos e tempo de recuperação inferior a cinco minutos (OKAMURA et al., 2010). Um dos anestésicos mais utilizados na piscicultura é a benzocaína pela segurança na aplicação tanto ao homem como para os peixes, tem custo baixo e é relativamente fácil de ser encontrado para a compra. Porém, é interessante ressaltar que o uso de anestésicos como MS-222, quanaidina, benzocaína e phenoxiethanol podem causar efeitos colaterais como perda de muco, irritação de brânquias e olhos (INOUE; SANTOS NETTO; MORAES, 2003).

Quando são realizados experimentos relacionados a anestésicos, é importante monitorar a condição corporal dos peixes. Esta condição geralmente é expressa por meio do fator de condição - um índice calculado a partir da razão entre o peso observado e o comprimento (LECREN, 1951). A condição corporal dos peixes pode levar a diferentes respostas frente aos anestésicos. As diferentes genéticas, idade e o meio também podem interferir nestes resultados (ROSS; ROSS, 2008). 
Diversos autores realizaram trabalhos com o objetivo de determinar a dose de anestesia (benzocaína) em várias espécies de peixes. De uma forma geral, as doses giram em torno de 100 a $200 \mathrm{mg} /$ litro de água, conforme Gomes et al. (2001) no tambaqui; Pharma e Croux (1990) no curimatã; Delbon (2006) na tilápia; Gimbo et al. (2008) no lambari (Astyanax altiparanae); BastosRamos, Gonçalves e Bacila (1998) no antarctic fish; Okamoto et al. (2009) no pampo (Trachinatus marginatos). Na carpa (Cyprinus carpio), Antunes et al. (2008) determinaram uma equação de predição, e estimaram que a cada aumento em $1 \mathrm{~g}$ de peso corresponde a um aumento de $0,16 \mathrm{mg} /$ litro de água na dose de benzocaína. O objetivo deste trabalho foi determinar a dose ótima de benzocaína em função do peso corporal através de uma equação de predição, em tilápias.

\section{Material e Métodos}

O experimento foi realizado no ano de 2009 na estação de piscicultura da Universidade Estadual de Londrina-Pr. O município localiza-se a $23^{\circ} 23^{\prime}$ de latitude sul e $51^{\circ} 11^{\prime}$ de latitude oeste, com altitude média de 566 metros. O clima, segundo a classificação de Köppen, é do tipo cfa, ou seja, subtropical úmido.

Foram utilizados 240 peixes (Oreochromis niloticus) divididos em seis etapas com 40 peixes cada. As médias de peso para cada etapa foram: 25,$64 ; 167,58 ; 186,12 ; 388,24 ; 518,19$ e 592,71. $\mathrm{O}$ intervalo entre cada etapa foi de dois meses para garantir a ausência de anestésico residual. Os peixes de mesma desova foram criados em tanque externo com superfície de $100 \mathrm{~m}^{2}$ e profundidade de $1,2 \mathrm{~m}$, e alimentados com ração comercial contendo 30\% PB e $2800 \mathrm{Kcal}$. Antes da anestesia foram capturados e colocados em tanques menores com capacidade para 1000 litros de água. Em seguida, foram capturados ao acaso e colocados individualmente em bacias de plástico contendo água e o anestésico nas concentrações a serem testados.
A temperatura da água foi mantida em torno de $23^{\circ} \mathrm{C}$ e ph ao redor de 7 . Em cada etapa foram realizados quatro tratamentos: 500, 700, 900, 1100 mg de benzocaína diluída em $20 \mathrm{ml}$ de etanol e depositado em bacias de plástico contendo 5 litros de água com fonte de ar. Caracterizando os tratamentos: $100 \mathrm{mg} / \mathrm{L}, 140 \mathrm{mg} / \mathrm{L}, 180 \mathrm{mg} / \mathrm{L} \mathrm{e}$ $220 \mathrm{mg} / \mathrm{L}$ de benzocaína por litro de água. O tempo de indução (TI) foi definido como o tempo em segundos entre a colocação do peixe no tanque e o desaparecimento dos movimentos operculares (estágio 6).

Após a indução anestésica, cada peixe foi mantido fora da água por 10 minutos para a realização da biometria. O peso corporal foi determinado em gramas e o comprimento corporal total em centímetros. Com os valores do peso corporal e o comprimento corporal total foi calculado o fator de condição alométrico, conforme Lecren (1951), através da formula:

$$
\mathrm{F}=\left(\mathrm{W} / \mathrm{L}^{\mathrm{b}}\right) .100
$$

Em que:

$\mathrm{W}=$ peso corporal $(\mathrm{g})$

$\mathrm{L}=$ comprimento corporal $(\mathrm{cm})$

$\mathrm{b}=$ coeficiente de regressão.

Em seguida os peixes foram colocados em um tanque com capacidade para 20 litros de água renovada continuamente, para permitir a sua recuperação representada pela volta dos movimentos operculares.

Em cada etapa foi determinada a dose mínima de benzocaína através do LRP (Linear Response Plateu) utilizando o aplicativo SAEG (UFV, 1983), em um modelo que incluiu dose e tempo de indução como variáveis. Ao final do experimento foi determinada a relação entre a dose de benzocaina $(\mathrm{mg} / \mathrm{l})$ pelo LRP e o peso corporal médio $(\mathrm{g})$ das tilápias em cada etapa. 


\section{Resultados e Discussão}

Na Tabela 1 são apresentadas as médias e erros padrão das características: tempo de indução, tempo de recuperação e o fator de condição conforme a dose de benzocaína.

Em relação ao tempo de indução pode-se afirmar que existe diferença entre as dosagens de benzocaina $(\mathrm{P}<0,05)$. Desta forma, a dose ideal foi de $180 \mathrm{mg}$. O tempo de recuperação não apresentou diferença estatística $(\mathrm{P}>0,05)$, o que concorda com Delbon (2006) que não encontrou diferenças entre os tempos de recuperação para tilápias submetidas à anestesia pela benzocaina.

Tabela 1. Médias e erros padrão das características: Tempo de indução (TI), Tempo de recuperação (TR) e fator de condição alométrico (FA), conforme a dose de benzocaina.

\begin{tabular}{cccc}
\hline Dose $(\mathrm{mg} / \mathrm{l})$ & TI $(\mathrm{s})$ & TR $(\mathrm{s})$ & FA \\
\hline 100 & $250,28 \pm 8,06 \mathrm{a}$ & $110,00 \pm 8,68$ & $2,58 \pm 0,033$ \\
140 & $149,40 \pm 5,28 \mathrm{~b}$ & $126,37 \pm 8,58$ & $2,56 \pm 0,039$ \\
180 & $115,24 \pm 6,45 \mathrm{c}$ & $120,40 \pm 9,24$ & $2,55 \pm 0,047$ \\
220 & $98,50 \pm 3,98 \mathrm{c}$ & $132,00 \pm 6,94$ & $2,69 \pm 0,058$ \\
\hline
\end{tabular}

Fonte: Elaboração dos autores.

$\mathrm{O}$ fator de condição não apresentou diferença ( $\mathrm{P}$ $>0,05)$, indicando que todos os peixes envolvidos no experimento estavam nas mesmas condições de manejo. $O$ fator de condição serviu também para mostrar que a anestesia pela benzocaina não interferiu no desenvolvimento normal dos peixes.

A condição que o peixe apresentou durante o experimento é um fator a ser levado em consideração, pois animais debilitados tendem a reagir com maior intensidade aos anestésicos. O fator de condição alométrico é calculado através do valor do b, e este pode assumir valores entre 2,5 a 4,0 (LECREN, 1951) ou 2,0 a 3,5 (ROYCE, 1972). Geralmente giram em torno de 3,0 (crescimento isométrico) e foi um indicativo do bom desenvolvimento dos peixes, o valor encontrado no presente trabalho e que serviu para o calculo do fator de condição alométrico foi de $\mathrm{b}=2,89$ sendo compatível a faixa de valores citados pelos autores acima, este valor foi significativo ( $\mathrm{P}$ $<0,05)$ indicando ser de crescimento alométrico negativo. Em todas as etapas o fator de condição alométrico variou pouco e não foi significativo ( $\mathrm{P}$ $>0,05)$.

Na tabela 2 são apresentados o peso corporal médio $(\mathrm{g})$, comprimento corporal médio $(\mathrm{cm})$, dose de benzocaína (mg/l), tempo de indução (TI-seg), determinado pelo LRP.

O valor da dose ótima de benzocaina determinada pelo LRP ficou na faixa entre 146 a $167 \mathrm{mg}$ de benzocaina/litro de água. Estes valores estão dentro da faixa de 100 a $200 \mathrm{mg}$ de benzocaina/litro de água (VETERINARY FORMULARY, 2008); Gomes et al. (2001) no tambaqui; Pharma e Croux (1990) no curimatã; Delbon (2006) na tilápia; Gimbo et al. (2008) no lambari (Astyanax altiparanae); BastosRamos, Gonçalves e Bacila (1998) no antarctic fish; Okamoto et al. (2009) no pampo (Trachinatus marginatos). Valores bem menores, entre 25 a 50 mg/l, são citados no AVMA (2007). 
Tabela 2. Pesos corporal médio (g); Comprimento corporal médio $(\mathrm{cm})$; Dose $(\mathrm{mg} / \mathrm{l})$ e Tempo de indução (TI);determinado pelo LRP.

\begin{tabular}{cccc}
\hline Pesos $(\mathrm{g})$ & Comp $(\mathrm{cm})$ & Dose/l & TI $(\mathrm{seg})$ \\
\hline 25,64 & 10,91 & 146,60 & 67,45 \\
167,58 & 20,19 & 155,95 & 76.33 \\
286,12 & 25,22 & 160,45 & 87,42 \\
388,24 & 28,59 & 167,00 & 108,14 \\
518,19 & 30,39 & 165,87 & 174,03 \\
592,71 & 32,13 & 164,00 & 139,80 \\
\hline
\end{tabular}

Fonte: Elaboração dos autores.

Okamura et al. (2010) avaliando tilápias de diferentes comprimentos sobre diferentes concentrações de benzocaína, observaram que para que o peixe permaneça sob sedação total, é necessário uma concentração de $190 \mathrm{mg} / \mathrm{L}$ de benzocaína, e este permanece 91 segundos sedado.

As doses utilizadas se mostraram seguras, indicando ausência de efeitos nocivos e mortalidade nos peixes utilizados no experimento. No entanto, neste experimento com os valores determinado pelo LRP foi possível estimar uma equação de predição, em que dentro de uma faixa de pesos de 25,64 a 592,71 , pode-se determinar qualquer dose ótima de anestésico que induza o estagio 6 em tilapia (Oreochromis niloticus). A média dos tempos de indução observados neste estudo foi de 47,45 a 174,03 segundos. Este valor está ligeiramente abaixo do intervalo de tempo (120 a 240 segundos) indicado por Olfert, Cross e Mcwilliam (1993) e dentro dos limites citado pela AVMA (2007) para anestesia, cirurgia, analgesia e cuidados pósanestésicos em peixes (60 a 180 segundos).
O presente trabalho relacionou a dose ótima com o peso obtendo a equação: Dose $=149,48+0,03183$ $\mathrm{x}$ Peso, $\mathrm{r}^{2}=0,73$ (Gráfico 1). Este resultado indica que um aumento no peso em $1 \mathrm{~g}$ corresponde a um aumento de 0,032 mg/l na dose de benzocaina para tilápias. Antunes et al. (2008) encontraram um valor $\mathrm{de} b=0,16$ indicando que $1 \mathrm{~g}$ de peso vivo em carpas resultou em um aumento na dosagem de anestésico em $0,16 \mathrm{mg} / \mathrm{litro}$ de água, sendo este valor superior ao encontrado no presente trabalho. Carneiro (2010) em experimento com transporte de matrinxã (Brycom cephalus), chegou à conclusão que o uso de benzocaína não reduziu o estresse causado pelo transporte, atuando inclusive como agente estressor adicional. No entanto, no presente trabalho, com o uso da benzocaína, não foi observado nenhum dos inconvenientes citados. Segundo os autores Olfert, Cross e Mcwilliam (1993) tem sido observada uma dose de segurança muito pequena entre a dose eficaz e a dose letal de benzocaina. Nas doses utilizadas neste experimento: 100, 140, 180 e $220 \mathrm{mg} / 1$, não foi constatado efeito colateral e óbito. Desta forma, as doses indicadas são seguras, concordando com Antunes et al. (2008) na carpa (Cyprinus carpio). 
Gráfico 1. Relação entre dose de benzocaína $(\mathrm{mg} / \mathrm{l})$ e peso médio $(\mathrm{g})$ determinada pelo LRP.

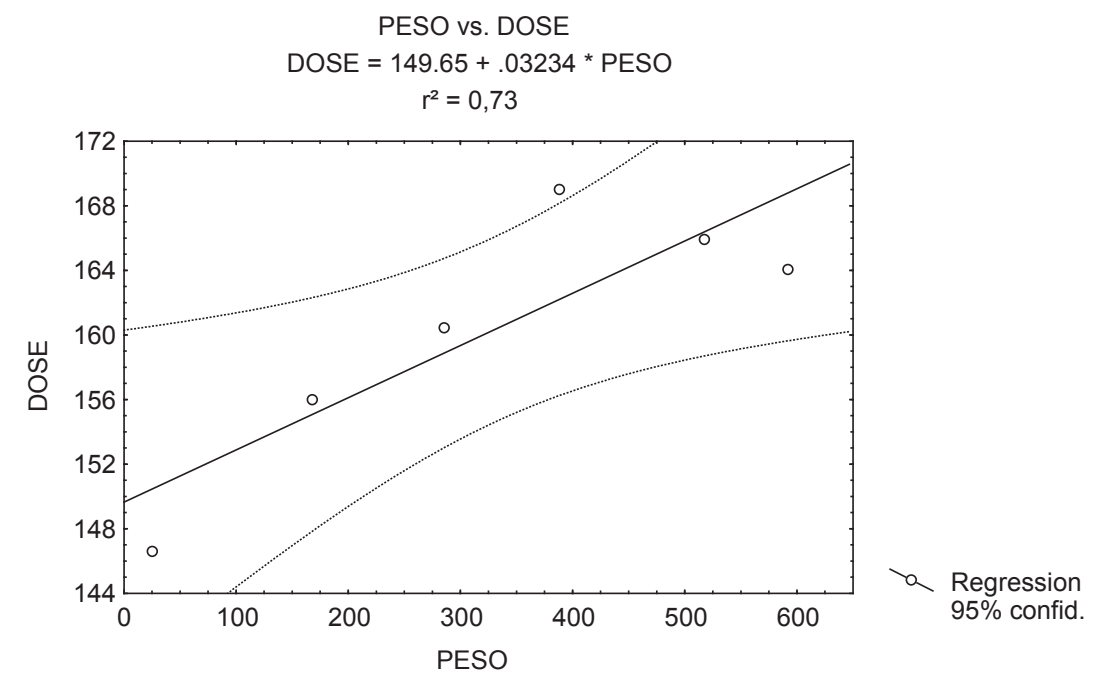

Fonte: Elaboração dos autores.

\section{Conclusões}

Os resultados permitem concluir que partindo de uma dose mínima de 146,60 e peso 25,64 um aumento no peso em $1 \mathrm{~g}$, corresponde a um aumento de $0,032 \mathrm{mg} / 1$ na dose de benzocaina para se obter a anestesia no estagio 6 em tilápias (Oreochromis niloticus).

\section{Referências}

ANTUNES, M. I. P. P.; SPURIO, R. S.; GODOI, D. A.; GRUMADAS, C. E. S.; ROCHA, M. A. Cloridrato de benzocaína na anestesia de carpas (Cyprinus carpio). Semina: Ciências Agrárias, Londrina, v. 29, n. 1, p. 151156,2008 .

AVMA guidelines on euthanasia. University of Michigan, jun., 2007. Disponível em: <http://www.avma.org/ issues/animal_welfare/euthanasia.pdf $>$. Acesso em: 28 out. 2008.

BASTOS-RAMOS, W. P.; GONÇALVES, N. M. F. M.; BACILA, M. Anesthesia and analgesia in Antartic fish; an experimental approuch. Archives of Veterinary Science, Curitiba, v. 3, n. 1, p. 95-100, 1998.

CARNEIRO, P. C. F. Estresse provocado pelo transporte e respostas fisiológicas do matrinxã Brycon cephalus (Teleostei: charicidae). 2010. Tese (Doutorado em Zootecnia) - Universidade Paulista Julio de Mesquita Filho, Jaboticabal.
DELBON, M. C. Ação da benzocaina e do óleo de cravo sobre parâmetros fisiológicos de tilápia (Oreochromis niloticus). 2006. Dissertação (Mestrado em Aqüicultura) - Universidade Estadual Paulista Julio Mesquita Filho, Jaboticabal.

GIMBO, R. Y.; SAITA, M. V.; GONÇALVES, A. F. N.; TAKAHASHI, L. S. Diferentes concentrações de benzocaína na indução anestésica do lambari do rabo amarelo (Astyanax altiparanae). Revista Brasileira de Saúde e Produção Animal, Salvador, v. 9, n. 2, p. 360357, 2008.

GOMES, L. C.; CHIPPARI-GOMES, A. R.; LOPES, N. P.; ROUBACH, R.; ARAUJO-LIMA, C. A. R. M. Efficacy of benzocaine as an anesthetic in juvenile tambaqui (Colossoma macroponum). Journal of the World Aquaculture Society, Baton Rouge, v. 32, n. 4, p. 426-431, 2001.

INOUE, L. A. K. A.; SANTOS NETTO, C.; MORAES, G. Clove oil as anaesthetic for juveniles of matrinxã (Brycon cephalus) (Gunther, 1869). Ciência Rural, Santa Maria, v. 33, n. 5, p. 943-947, 2003.

LECREN, E. D. The length-weight relationship and seasonal cycle in gonad weight and condition in the perch (Perca fluviatis). Journal of Animal Ecology, Oxford, v. 20, p. 201-219, 1951.

OKAMOTO, M. H.; TESSER, M. B.; LOUZADA, L. R.; SANTOS, R. A.; SAMPAIO, L. A. Benzocaína e eugenol como anestésicos para juvenis de pampo (Trachinatus marginatus). Ciência Rural, Santa Maria, v. 39, n. 3, p. 866-870, 2009. 
OKAMURA, D.; ARAÚJO, F. G.; ROSA, P. V.; FREITAS, R. T. F.; MURGAS, L. D. S.; CESAR, M. P. Influência da concentração de benzocaína e do comprimento dos peixes na anestesia e na recuperação de tilápias-do-nilo. Revista Brasileira de Zootecnia, Viçosa, v. 39, n. 5, p. 971-976, 2010.

OLFERT, E. D.; CROSS, B. M.; MCWILLIAM, A. A. Guide to the care and use of experimental animals. 2. ed. Ottawa: Canadian Council on Animal Care, 1993. 298 p.

PHARMA, S. E.; CROUX, M. J. Benzocaine(ethyl -p-aminobenzoate) as an anesthetic for Prochilodus lineatus, Valenciunes (pices curimatidae). Journal of Applied Ichthyology, v. 6, n. 3, p. 189-192, 1990.

ROSS, L. G.; ROSS, B. Anaesthetic and sedative techniques for aquatic animals. 3. ed. Oxford: Blackwell, 2008. 222 p.
ROUBACH, R.; GOMES, C. L. O uso de anestésico durante o manejo de peixes. Panorama da Aqüicultura, Rio de Janeiro, v. 11, n. 6, p. 37-40, 2001.

ROYCE, W. F. Introduction to the fishery science. New York: Academic Press, 1972. 351 p.

THURMON, J. C.; TRANQUILLI, W. J.; BENSON, G. J. Anesthesia of wild, exotic and laboratory animals. In: THURMON, J. C.; TRANQUILLI, W. J.; BENSON, G. J. (Ed.). Lumb and Jones, veterinary anesthesia. 3. ed. Baltimore: Williams \& Wilkins, 1996. p. 686-735.

UNIVERSIDADE FEDERAL DE VIÇOSA - UFV. SAEG. Manual de utilização do programa - sistema para análise estatística e genética. Viçosa: Imprensa Universitária, 1983.

VETERINARY FORMULARY. 2008. Disponível em: $<$ http//www.ahc.umn.edu/rar>. Acesso em: 28 out. 2008. 
